\title{
Tecnologias sociais, formação docente e comunidades de aprendizagem: um desafio contemporâneo
}

\author{
Graziella Rollemberg1, Mário André de Freitas Farias ${ }^{1}$
}

${ }^{1}$ Programa de Mestrado Profissional em Educação Profissional e Tecnológica - Instituto Federal de Sergipe - Aracaju - SE - Brasil

graziella.rollemberg141@academico.ifs.edu.br,mario.andredifs.edu.br

\begin{abstract}
This article addresses the challenge of overcoming technocentrism through the cooperative production of social innovations with the support of collaborative computing resources. The paper seeks to show how participatory construction, integrating different agents, of a systematic methodology for the production of Social Technologies and its application to teacher education through educational technology products that promote the formation of learning communities, can constitute a new model of generating sustainable solutions in search of social inclusion.

Resumo. Este artigo aborda o desafio de superar do tecnocentrismo por meio da produção cooperativa de inovações sociais com apoio de recursos computacionais colaborativos. $O$ trabalho procura mostrar como a construção participativa, integrando diversos agentes, de uma metodologia sistemática de produção de Tecnologias Sociais e sua aplicação à formação docente por meio de produtos de tecnologia educacional que promovam a formação de comunidades de aprendizagem, podem constituir-se em um novo modelo de geração de soluções sustentáveis em busca da inclusão social.
\end{abstract}

\section{Introdução}

No contexto da sociedade contemporânea, um novo paradigma de inovação delineia-se, fazendo com que a inovação social seja cada vez mais importante. Esse processo traz em seu bojo a discussão sobre os novos papéis que as ciências e a comunidade científica podem desempenhar na análise e modelagem da inovação social e sobre a necessidade de desenvolver teorias e práticas integradas à inovação sócio tecnológica, na qual a inovação social não seja apenas mero resultado da inovação técnica, mas sim produto da reflexão, do trabalho e da ação de pesquisadores de múltiplas áreas científicas, como a Educação, a Computação, as Ciências Sociais, entre várias outras.

Um dos pontos importantes a serem discutidos sob uma perspectiva integrada e integradora dos saberes e das práticas, tanto técnico-científicas quanto sociais, é o conceito de tecnologia e suas relações com a ciência, a educação, a cultura e com os diversos aspectos da sociedade. Segundo Bazzo (1998), a abordagem crítica da tecnologia posiciona "uma reação social crítica ao cientificismo e à tecnocracia" e busca compreender a dimensão social da ciência e da tecnologia em relação aos fatores de natureza social, política e econômica, responsáveis pela mudança científica e tecnológica, e, também, no que diz respeito às repercussões éticas, ambientais ou culturais dessa mudança.

Diferentes perspectivas teóricas em relação ao fenômeno da aceleração tecnológica foram elaboradas a partir da intensificação dos processos técnico-científicos 
na contemporaneidade, de um lado, a concepção de que o progresso crescente da ciência e da tecnologia constitui-se determinante para o desenvolvimento econômico e social, além de possuir natureza neutra e não normativa e, de outro, a perspectiva fundada nas indeterminações que rondam o futuro da sociedade, moldada pelos sistemas tecnocientíficos contemporâneos, perspectiva que questiona o suposto papel determinante e neutro da ciência e da tecnologia [Garcia, 2007].

O modelo da cadeia linear da inovação tecnológica convencional, inspirada no trabalho de Schumpeter (1961), supõe que a pesquisa científica é seguida pela tecnológica, que a pesquisa tecnológica, por sua vez, produz o desenvolvimento econômico, e este possibilita o desenvolvimento social.

Inovações, em tal acepção, seriam então processos por meio dos quais novas ideias, objetos e práticas são criados, desenvolvidos ou reinventados [Crossan; Apaydin, 2010]. Essa perspectiva, portanto, relaciona diretamente o avanço ou atraso das sociedades ao nível de sofisticação tecnológica que possuem.

Sob perspectiva oposta, as tecnologias são vistas como construídas socialmente, isto é, como sendo formadas por conhecimentos e produtos humanos, e refletem os valores e as contradições das sociedades que as engendram. Sob essa visão, questiona-se se a tecnociência e as tecnologias convencionais responderiam efetivamente às inúmeras carências e problemas sociais e econômicos da sociedade.

Um viés teórico que emerge como de grande importância na busca de respostas a tal questionamento é o de Álvaro Vieira Pinto, que faz uma análise crítica da tecnologia na sociedade atual e de sua relação com a educação, enfocando especialmente o problema do tecnocentrismo [Pinto, 2005]. As análises de Vieira Pinto sobre as ideologizações da tecnologia apontam para os interesses que podem estar por trás da tecnicização do campo pedagógico, com sérias críticas à forma hegemônica, também nesse campo, de se compreender a tecnologia.

\section{Desafios em diversidade e inclusão em ecossistemas de educação}

A inclusão sob a perspectiva social deve levar em conta as particularidades das comunidades locais e dos atores sociais dos ecossistemas de educação que lhes são próprios. Nesse sentido, é de grande importância empreender ações que possam desconstruir a crença de que a tecnologia pode resolver tudo, o que seria, segundo Vieira Pinto, uma forma equivocada de percebê-la, pois ela não é produtora do humano, mas sim produto do ser humano. Sendo assim, a técnica só pode beneficiar toda a sociedade, sem exclusões, se for encarada como construção possível à qualquer comunidade, autogestionada e com apoio de parceiros externos, em busca das soluções efetivamente adequadas aos problemas locais, tais como os educacionais.

Nesse cenário, surge a possibilidade de pensar a tecnologia como uma dimensão da cultura, resgatando para o campo pedagógico uma compreensão histórica que situa a tecnologia como uma atividade humana subordinada a outras dimensões humanas, e não superior a elas [Dagnino, 2014], traduzida no que no Brasil tem sido denominado de Tecnologias Sociais (TSs). Segundo a Rede de Tecnologia Social - RTS, Tecnologia Social compreende produtos, técnicas ou metodologias, reaplicáveis, desenvolvidas na interação com a comunidade e que devem representar efetivas soluções de transformação social. [Otterloo, 2009]. 
As tecnologias sociais, exemplos significativos de inovação social, podem incorporar, em suas ações pedagógicas no domínio educativo, os conceitos de inteligência coletiva [Levy, 1999] e de aprendizagem cooperativa regida pelas novas relações com o saber [Charlot, 2016], poderia ser extrapolado para comunidades presenciais, para uma práxis comunitária com o suporte de novas ferramentas computacionais [Soffner, 2014].

Surge, então, como desafio relevante para a próxima década a superação do tecnocentrismo por meio da produção colaborativa de inovações sociais com apoio de recursos computacionais aplicados de forma também inovadora à formação docente.

A construção de TSs por meio da cooperação e integração de diversos agentes, apoiados por recursos computacionais colaborativos e aplicados à formação docente é uma forma de construção de soluções que podem promover a inclusão social.

Entretanto, para enfrentar tal desafio, é preciso construir vias práticas de implementação de novos itinerários de formação docente para a produção e transferência de tecnologias sociais, ancoradas em uma pedagogia que efetivamente considere as novas relações com o saber e utilize sistemas e ferramentas computacionais colaborativas, o que representa uma alternativa de abordagem pedagógica em relação ao paradigma predominante no uso de tecnologias na educação.

O campo pedagógico tem sido fértil em adaptações passivas e acríticas de projetos tecnológicos, os quais, muitas vezes, em vez de meios, tornam-se fins, como no caso das propostas que permanecem centradas na inserção das novas tecnologias na escola sob a perspectiva tecnicista, focada no acesso, ao invés de contribuir para o processo de socialização das aprendizagens e de produção colaborativa de conhecimentos na comunidade escolar.

A hipótese de que oferta de equipamentos, disponibilização de ferramentas tecnológicas e treinamentos operacionais favoreceriam por si só o desenvolvimento de competências digitais foi contestada pelo relatório Estudantes, Computadores e Aprendizado: Fazendo a Conexão, OECD (2015), realizado no âmbito do Pisa (Programa Internacional de Avaliação de Estudantes) da OCDE (Organização para a Cooperação e Desenvolvimento Econômico), que resultou do primeiro estudo da organização analisando as competências de alunos de 31 países do mundo na área digital.

O relatório revelou, com o a análise de exemplos como o da Austrália, que tem maior taxa mundial de computadores por aluno, mas não está entre os países com melhor desempenho, que o acesso ao computador só contribui para formação de competências digitais dos alunos quando os professores recebem formação ou têm experiência em educação para as mídias.

\section{A importância e os benefícios de se buscar solução para o Desafio}

Os benefícios de conseguir superar o tecnocentrismo por meio da produção cooperativa de inovações sociais apoiado por recursos computacionais colaborativos de formação continuada de professores são:

- Enfrentar as resistências de docentes e outros membros das comunidades escolares em construir e aplicar práticas pedagógicas apoiadas em modelos inovadores de uso das tecnologias aplicadas à educação;

- Promover o diálogo e a cooperação entre a comunidade acadêmica, as organizações do terceiro setor envolvidas com o desenvolvimento de inovações sociais e com tecnologias aplicadas à educação e as comunidades externas em geral; 
- Mobilizar docentes, discentes, e outros membros da comunidade escolar e das populações do entorno das instituições educacionais para discutir e construir conjuntamente, de modo sistematizado e reaplicável, soluções sustentáveis para problemas sociais, culturais e ambientais relevantes para as comunidades locais;

- Apoiar efetivamente as práticas pedagógicas de docentes na geração e adaptação de soluções técnicas e tecnológicas às demandas sociais e peculiaridades regionais, promovendo a produção, o desenvolvimento e a transferência de tecnologias sociais relevantes para o desenvolvimento local.

- Oportunizar o acesso, às comunidades locais, de conhecimentos científicos e metodológicos importantes para subsidiar a produção de TSs e, à comunidade acadêmica, às comunidades escolares e aos parceiros de instituições não governamentais, de conhecimentos tácitos, experiências e práticas das comunidades locais;

- Favorecer um intercâmbio efetivo de saberes e práticas no sentido da produção conjunta de TSs que promovam a inclusão social de modo adequado a cada comunidade e adaptável a outras comunidades com problemas e perfis sócio culturais, econômicos e ambientais semelhantes.

\section{Formas de suscitar colaborações para trabalhar no desafio}

Segundo Mulgan et al. (2007), para o sucesso das inovações sociais é essencial contar com o trabalho integrado, em cooperação, de vários agentes, internos e externos à comunidade, entre os quais os integrantes das comunidades científicas, instituições de ensino, organizações do terceiro setor etc.

É preciso considerar expectativas, contribuições e o conjunto de interesses de todos os integrantes dos processos educativos, comunicativos e sociais, criando um espaço relevante para que os sujeitos atuem efetivamente na construção social e na transformação de sua realidade. Essa noção complementa a de inclusão em ecossistemas de educação, tomado aqui como os distintos ambientes de produção da informação, do conhecimento, das identidades, e de práticas sócio - culturais, nos quais várias linguagens possam ser trabalhadas e uma diversidade de sujeitos possa interagir de maneira articulada [Candau, 2007].

Por meio do levantamento preliminar sobre projetos recentes de organizações não governamentais que atuam na área educacional no Brasil, no sentido de inovar as abordagens pedagógicas da formação docente, foi identificada e analisada a proposta de plataforma digital educativa Synapse, desenvolvida pelo Instituto de Pesquisas em Tecnologia e Inovação (IPTI), uma Organização Social que se dedica ao desenvolvimento de TSs nas áreas de educação, saúde pública e economias criativas, no sentido de produzir, aplicar e validar um guia metodológico interativo, construído e aplicado por meio de comunidades de aprendizagem, para apoio à formação docente em produção, transferência e reaplicação de Tecnologias Sociais.

A plataforma foi desenvolvida no bojo de um projeto de Tecnologia Social mais amplo aplicado à educação, é um exemplo promissor da inovação no uso de recursos digitais na formação de professores e no apoio às práticas pedagógicas. A plataforma tem por objetivo diagnosticar e treinar individualmente os alunos do primeiro ciclo do Ensino Fundamental da rede pública de ensino de Sergipe em suas dificuldades de aprendizagem por meio de sequências didáticas de atividades sobre Linguagem e Matemática. 
O IPTI demonstrou interesse em uma parceria com pesquisadores e docentes do Instituto Federal de Sergipe - Campus Lagarto para o desenvolvimento pioneiro de uma metodologia sistemática de formação em TSs, com critérios sócio técnicos efetivos para a produção e manutenção de TSs, que poderá ser por elas aplicada em seus projetos. A parceria foi firmada no sentido de promover a colaboração de instituições de ensino e pesquisa na área de tecnologias aplicadas à educação para auxiliar no desenvolvimento produtos educacionais que possam apoiar a formação docente na metodologia de produção e transferência de TSs é também fundamental.

A parceria pode prover o suporte necessário ao itinerário de formação docente apoiado por recursos computacionais colaborativos gerando comunidades de aprendizagem com o uso inovador de recursos interativos síncronos e assíncronos nos contextos de ensino e aprendizagem e atendendo às expectativas de melhoria das dinâmicas de produção e divulgação de conhecimentos em ecossistemas de educação.

\section{Potenciais dificuldades atuais e vindouras inerentes ao desafio}

As dificuldades que se anunciam para o enfrentamento do desafio passam por:

- Desconhecimento, por parte dos docentes e demais membros das comunidades escolares e dos próprios agentes das comunidades locais sobre o que é esse tipo específico de inovação social, a Tecnologia Social, sobre seu caráter eminentemente participativo e voltado para soluções práticas, reaplicáveis e sustentáveis nas comunidades e sobre a necessidade de seguir etapas e critérios sistemáticos para sua produção e transferência. Várias instituições de ensino e pesquisa e organizações do terceiro setor atuam em projetos de intervenção na realidade social que, muitas vezes, acabam sendo pouco efetivos ou descontinuando-se ao longo do tempo, pois não seguem etapas ou critérios sócio técnicos sistemáticos, o que resulta, também, em pouca escala de adaptação e reaplicação à realidades semelhantes;

- Ausência, em grande parte das propostas de formação de professores da dimensão cooperativa ou colaborativa das aprendizagens informais que vêm se desenhando nos últimos anos, e que são tão importantes para a formação das comunidades de aprendizagem e de intervenção na realidade necessárias à produção e implementação de TSs. A resistência de parte da comunidade escolar em relação às práticas inovadoras em geral é também componente desse tipo de dificuldade inerente ao desafio proposto;

- Dificuldade que as instituições de ensino e pesquisa tem tido em promover um diálogo vivo e próximo com a realidade local e regional com um olhar mais criterioso em busca de soluções para a realidade de exclusão, de mobilização e de manutenção de comunidades de aprendizagem envolvendo docentes, discentes, comunidade escolar e as populações locais, tantas vezes excluídas das instituições educacionais formais, e de soluções sustentáveis às demandas de suas comunidades.

\section{Ações que devem ser tomadas para se enfrentar o desafio em um período de 10 anos}

Um primeiro passo no caminho de solucionar o desafio proposto, cujas etapas traduzemse nas seguintes ações a serem tomadas na próxima década: 
- Pesquisas bibliográficas sobre características, critérios de produção, transferência e adequação sócio-técnica de TSs sobre experiências práticas já implementadas no Brasil e análise documental de relatórios internos, registros, relatos e relatórios dos pesquisadores e agentes de Organizações Não Governamentais (ONGs) envolvidas em projetos de TSs aplicadas com sucesso a comunidades brasileiras;

- Produção, com a parceria de ONGs atuantes na área de TSs e de docentes de contextos educacionais formais, de um guia metodológico digital interativo, aliada ao estabelecimento de comunidades de aprendizagem colaborativa com recursos síncronos e assíncronos para produção e reaplicação de TSs

- Formação de docentes em TSs por meio do guia metodológico digital interativo, aliada ao estabelecimento de uma comunidade de aprendizagem colaborativa.

- Avaliação do processo de formação visando eventuais reorientação de estratégias e melhoria do guia e das dinâmicas de aprendizagem;

- Ampliação do processo formativo com a incorporação de agentes sociais à comunidade de aprendizagem e aplicação das etapas previstas para a produção cooperativa de Tecnologia Social na comunidade local visando promover a inclusão social e a solução sustentável de problemas da população;

- Avaliação de todo o arco do processo com o objetivo de aprimorá-lo e eventualmente reaplica-lo em outras comunidades da região com perfis semelhantes.

\section{Considerações finais}

Pode-se considerar consenso entre os pesquisadores da área que, para garantir pluralidade, inclusão e participação social no contexto contemporâneo da sociedade da informação e especificamente nos ecossistemas educativos, é essencial oferecer a todos os cidadãos, sobretudo às novas gerações, não apenas acesso às novas tecnologias e mídias, mas também os meios para compreender as informações disponíveis de forma crítica e produzir informações e saberes de modo autônomo. Os sistemas educacionais têm um papel central nesse processo, mas para efetivamente educar para as mídias em um sentido amplo e abrangente, necessitam do apoio do poder público e de organizações da sociedade civil.

As tecnologias sociais, segundo Soffner (2014) são o resultado de projetos na área da pedagogia social entendida como uma ciência que produz, por meios de métodos, técnicas e soluções para problemas encontrados pelas pessoas no sentido de buscar a melhoria da qualidade de vida dos indivíduos e grupos, bem como o desenvolvimento educativo integral das pessoas envolvidas na transformação social do ambiente ao qual se aplica, incorporando também a noção de tecnologia educativa.

A difusão de projetos inovadores de formação continuada de docentes sob a perspectiva da mídia-educação pode favorecer a produção colaborativa de conhecimentos, opiniões e saberes nos meios digitais e transformar o cenário da aplicação das novas tecnologias em ecossistemas educacionais respeitando as especificidades locais.

A associação de propostas inovadoras de desenvolvimento local sustentável como as Tecnologias Sociais, às perspectivas abertas pela mídia-educação, quando aplicada aos processos de formação docente, pode constituir um novo modelo de construção coletiva de saberes baseados nas práticas pedagógicas dos professores e em suas demandas locais. 
Nesse sentido, o desafio da construção participativa de uma metodologia sistemática de produção de TSs e sua aplicação à formação docente por meio de um guia digital interativo construído e aplicado coletivamente por meio comunidades de aprendizagem ancoradas em recursos interativos podem constituir-se em um novo modelo de geração de soluções sustentáveis em busca da inclusão social e educacional.

As tecnologias sociais educativas aliadas ao uso das novas tecnologias da informação sob a perspectiva da mídia educação podem se constituir em um meio privilegiado de construção da autonomia social necessária aos avanços locais no âmbito dos ecossistemas de educação, constituindo uma efetiva práxis comunitária educativa com o suporte da tecnologia, em uma apropriação positiva da lógica das redes colaborativas virtuais de aprendizagem no sentido de articular diversos agentes na solução de problemas das comunidades locais. Afinal, como afirmam Davidson e Goldberg (2009), tecnologia não é revolução, mas o potencial para a aprendizagem compartilhada, interativa e colaborativa.

\section{Referências bibliográficas}

Araújo, R. O. A. e Cândido, G. A. (2017). Sistema de Indicadores para Diagnóstico, Monitoramento e Avaliação de Tecnologias sociais: Proposição de uma metodologia. Caracas: Revista Espacios, Vol. 38 ( $\mathrm{N}^{\mathrm{o}}$ 02), p. 18

Bazzo, W. A. (1998) Ciência, tecnologia e sociedade: o contexto da educação tecnológica. Florianópolis: Ed. da UFSC.

Candau, Vera Maria. (2007) "Construir ecossistemas educativos - reinventar a escola”. In: CANDAU, Vera Maria (org.). Reinventar a escola. 5. Ed. Petrópolis, RJ: Vozes, p.11 - 16 .

Charlot, B. (2016) Da relação com o saber às práticas educativas. São Paulo: Cortez Editora.

Crossan, M. M.; Apaydin, M. A (2010). Multi-Dimensional Framework of Organizational Innovation: A Systematic Review of the Literature. Journal of Management Studies, v. 47 n. 6, p. 1154-1191, Sept. (Tradução livre dos autores).

Dagnino, R. (2014) Tecnologia Social: contribuições conceituais e metodológicas [online]. Campina Grande: EDUEPB.

Davidson, C. N. e Goldberg, D. (2009). The Future of Learning Institutions in a Digital Age. CAMBRIDGE: MIT Press. Disponível em: [http://mitpress.mit.edu/9780262513593]. Acesso: 02 mar. 2018.

Garcia, J. C. D. (2007). Uma Metodologia de Análise das Tecnologias Sociais: as Tecnologias Sociais. Trabalho apresentado no XII Seminário Latino-Ibero-americano de Gestión Tecnológica - ALTEC 2007. Buenos Aires, Set.

Lévy, P. (1999). Cibercultura. São Paulo: Editora 34 Ltda.

Mulgan, G. (2006); The process of social innovation. innovations, v. 1, n. 2.

Pinto, A. V. (2005). O conceito de tecnologia. Rio de Janeiro: Contraponto.

Schumpeter J. A. (1961). Capitalismo, Socialismo e Democracia. Editado por George Allen e Unwin Ltd., traduzido por Ruy Jungmann). — Rio de Janeiro: Editora Fundo de Cultura. 
Soffner, R. K. (2014). Tecnologias sociais e a educação para a práxis sócio comunitária. Série-Estudos. Campo Grande, n. 37, p. 317, jan./jun.

Oecd, Students, Computers and Learning: Making the Connection (2015). Pisa, OECD Publishing. Disponível em: http://www.keepeek.com/Digital-Asset Management/oecd/education/students-computers-andlearning_9789264239555en\#page3 Acesso: 21 Abr. 2018.

Otterloo, A. [et al.] Tecnologias Sociais: caminhos para a sustentabilidade (2009). Brasília/DF: s.n. 